\title{
Portal Vein Embolization before Extended Hepatectomy for Biliary Cancer: Current Technique and Review of 494 Consecutive Embolizations
}

\author{
Tomoki Ebata Yukihiro Yokoyama Tsuyoshi Igami Gen Sugawara \\ Yu Takahashi Masato Nagino \\ Division of Surgical Oncology, Department of Surgery, Nagoya University Graduate School of Medicine, \\ Nagoya, Japan
}

\section{Key Words}

Portal vein embolization - Cholangiocarcinoma .

Gallbladder cancer $\cdot$ Hepatectomy

\begin{abstract}
Backgrounds: Portal vein embolization (PVE) has been widely applied before extended hepatectomy; however, its clinical utility for patients with biliary cancer has not been fully addressed. Methods: Between 1991 and 2010, 494 patients with cholangiocarcinoma $(n=353)$ or gallbladder cancer $(n=141)$ underwent PVE before extended hepatectomy. PVE was performed by a transhepatic ipsilateral approach using fibrin glue or absolute ethanol with steel coils. Surgical outcomes of this cohort were retrospectively reviewed. $\boldsymbol{R e}$ sults: PVE-related complications requiring interventions were found in $3(0.6 \%)$ of the 494 patients; no patient died of these complications. Among the 494 patients, 122 (24.7\%) did not undergo subsequent hepatectomy. The unresectability rate was significantly higher in patients with gallbladder cancer than in those with cholangiocarcinoma [43.2\% $(61 / 141)$ and $17.3 \%(61 / 353)$, respectively, $p<0.001]$. The remaining 372 patients underwent hepatectomy, and 24 (6.5\%) died of postoperative complications [13 of $80(16.3 \%)$ with gallbladder cancer vs. 11 of 292 (3.8\%) with cholangio-
\end{abstract}

carcinoma, $\mathrm{p}<0.05]$. The overall survival for patients with cholangiocarcinoma was significantly better than that for patients with gallbladder cancer, where the 5 -year survival rate was 39 and $23 \%$, respectively $(p<0.001)$. Thirty-six patients with cholangiocarcinoma and 10 patients with gallbladder cancer survived more than 5 years after extended surgery. Conclusion: PVE can be performed safely in patients with cholestatic liver, and it has a potential benefit for patients with advanced biliary cancer who are to undergo extended, difficult hepatectomy.

Copyright $\odot 2012$ S. Karger AG, Basel

\section{Introduction}

Portalvein embolization (PVE), introduced by Makuuchi and Kinoshita in 1980 [1, 2], can induce hypertrophy of the non-embolized lobe; thus, it has the potential to enhance the safety of extended hepatectomy [3]. At present, PVE is being used as a standard preoperative management in patients with inadequate liver functional reserve. Although the clinical utility of PVE before extended hepatectomy has been shown in patients with underlying liver disease, it has not been fully addressed for patients with cholestatic liver. We have used this radio-

\section{KARGER}

Fax +4161306 1234 E-Mail karger@karger.ch www.karger.com
(C) 2012 S. Karger AG, Basel

$0253-4886 / 12 / 0291-0023 \$ 38.00 / 0$

Accessible online at:

www.karger.com/dsu
Prof. Masato Nagino, $\mathrm{MD}, \mathrm{PhD}$

Division of Surgical Oncology, Department of Surgery

Nagoya University Graduate School of Medicine

65 Tsurumai-cho, Showa-ku, Nagoya 466-8550 (Japan)

Tel. +81 52744 2217, E-Mail nagino@ @ed.nagoya-u.ac.jp 
logic intervention for biliary cancer since 1990, developed a novel technique that included an ipsilateral approach [4] and trisegment PVE [5-7], and investigated the changes in volume [8], function [9], and hemodynamics [10-12].

We have previously reported the surgical outcome and long-term results of 240 PVEs between 1991 and 2005, concluding that PVE has a potential benefit for patients with advanced biliary cancer who are to undergo extended, complex hepatectomy [13]. As 5 years have passed since this report and our experience with PVE has doubled, we herein describe our current technique and updated results of PVE before extended hepatectomy for biliary cancer.

\section{Patients and Methods}

\section{Patients}

Between January 1991 and December 2010, 494 patients with cholangiocarcinoma $(n=353)$ or gallbladder cancer $(n=141)$ underwent PVE before scheduled extended hepatectomy at the First Department of Surgery, Nagoya University Hospital. Of the 494 patients, 240 patients between 1991 and 2005 overlapped with the subjects in our previous report [13]. The study group reported herein comprised 284 men and 210 women, with an average age of $63 \pm 11$ years. Of the 494 patients, $386(78.1 \%)$ had obstructive jaundice at initial presentation and then underwent preoperative biliary drainage before PVE. Another 40 patients without jaundice also underwent biliary drainage to relieve cholangitis and/or to determine the extent of cancer.

\section{Portal Vein Embolization}

In principle, PVE was indicated when a future liver remnant was estimated to be less than $40 \%$ and was performed 2-4 weeks before a scheduled hepatectomy. In jaundiced patients, the intervention was performed after the serum total bilirubin concentration had decreased to less than $5 \mathrm{mg} / \mathrm{dl}$ following biliary drainage.

In our service, hepatobiliary surgeons, not radiologists, perform PVE using a percutaneous transhepatic ipsilateral technique [4] in a radiologic suite. Under local anesthesia with $1.0 \%$ lidocaine and conscious sedation with intravenous administration of pentazocine and midazolam, the portal branch of the sector to be resected was punctured (ipsilateral approach), thereby minimizing the risk of vascular injury in the future remnant liver. The right anterior sectorial portal branch was routinely targeted for access in any type of PVE (right PVE and right or left trisegment PVE), unless it was obstructed by tumor involvement. First, a clear portogram of at least 3 different projections was obtained, e.g. supine position, right anterior oblique projection, and right anterior oblique projection with caudally tilting $20^{\circ}$, to confirm the individual anatomy of the portal system [14]. The third projection often provided the best visualization of the root of the right anterior/posterior portal vein and the left portal vein.

The embolic material fibrin glue (Bolheal; Astellas Pharma Inc., Tokyo, Japan) mixed with iodized oil (Lipiodol Ultra-Fluide;
Terumo, Tokyo, Japan) was used before the year $2000(\mathrm{n}=131)$, whereas absolute ethanol [15] with steel coils (MReye Embolization Coil; Medicos Hirata, Osaka, Japan) was used after 2001 ( $\mathrm{n}=$ 363), because the Prefectural Insurance System prohibited the use of glue for PVE due to its high price. Absolute ethanol permeates into the peripheral portal system, provokes endothelial damage/ thrombus, and ends in occlusion of the portal vein. Ethanol has no radio-opacity, which carries a risk of unintended back flow to the portal veins in the non-embolized liver. The specific gravity of this agent is lighter than blood, which potentially yields an unequal embolization in the targeted vein. To avoid these adverse complications, $0.5-2.0 \mathrm{ml}$ ethanol was slowly administered into each targeted portal branches by selective catheterization. This injection was continued until stasis or near-stasis of the flow was achieved. Finally, embolization steel coils were appropriately placed to consolidate the permanent and complete embolization. At least a 1-cm-long proximal segment was to remain unembolized to facilitate the division and closure of the portal vein at surgery (fig. 1).

For this purpose, right PVE was routinely performed using the following sequence. A 4-Fr reversed curve catheter (CJ-1, Terumo Clinical Supply, Gifu, Japan) was chosen for easy manipulation in the right branches, given the severe angulation of the right portal tree with the ipsilateral approach. First, the catheter was advanced into the right posterior portal vein, and then the posterior trunk was embolized. When the inferior (P6) and the posterior branch (P7) of the right posterior sector were independently bifurcated without formation of the common trunk, both were embolized separately. Second, inferior branches (P5) proximally ramifying from the right anterior portal vein were separately embolized. Third, the superior branches (P8) of the right anterior sectors were also embolized. Finally, the puncture site was closed with steel coils to prevent intra-abdominal bleeding.

\section{Trisegment PVE}

The ipsilateral approach through the right anterior portal vein allowed us to perform a right or left trisegment PVE [5-7]. A right trisegment PVE needed an embolization of the left medial segment branch (P4) in addition to the right PVE. Because this extended intervention significantly improved hypertrophy of the left lateral segment $(\mathrm{S} 2+3)$ compared with the right PVE alone [6], it was routinely attempted as far as technically possible. Catheterization of $\mathrm{P} 4$ is often technically difficult, because some $\mathrm{P} 4 \mathrm{~s}$ are thin, multiple, and steep angled [16]. A 2.2-Fr microcatheter (Progreate $\beta$; Terumo Medical Corporation, Tokyo, Japan) coaxially through a 4-Fr guiding catheter or an angiographic catheter manually adjusted according to the angulation is useful for safe P4 embolization. Also, a left trisegment PVE (embolization of the left and the right anterior portal vein) can be performed with similar techniques (fig. 2).

\section{Statistics}

Results were expressed as mean \pm SD. Categorical data were compared using the $\chi^{2}$ test. Postoperative survival was calculated using the Kaplan-Meier method. Differences in survival curves were compared using the log-rank test. $p<0.05$ was considered statistically significant. 

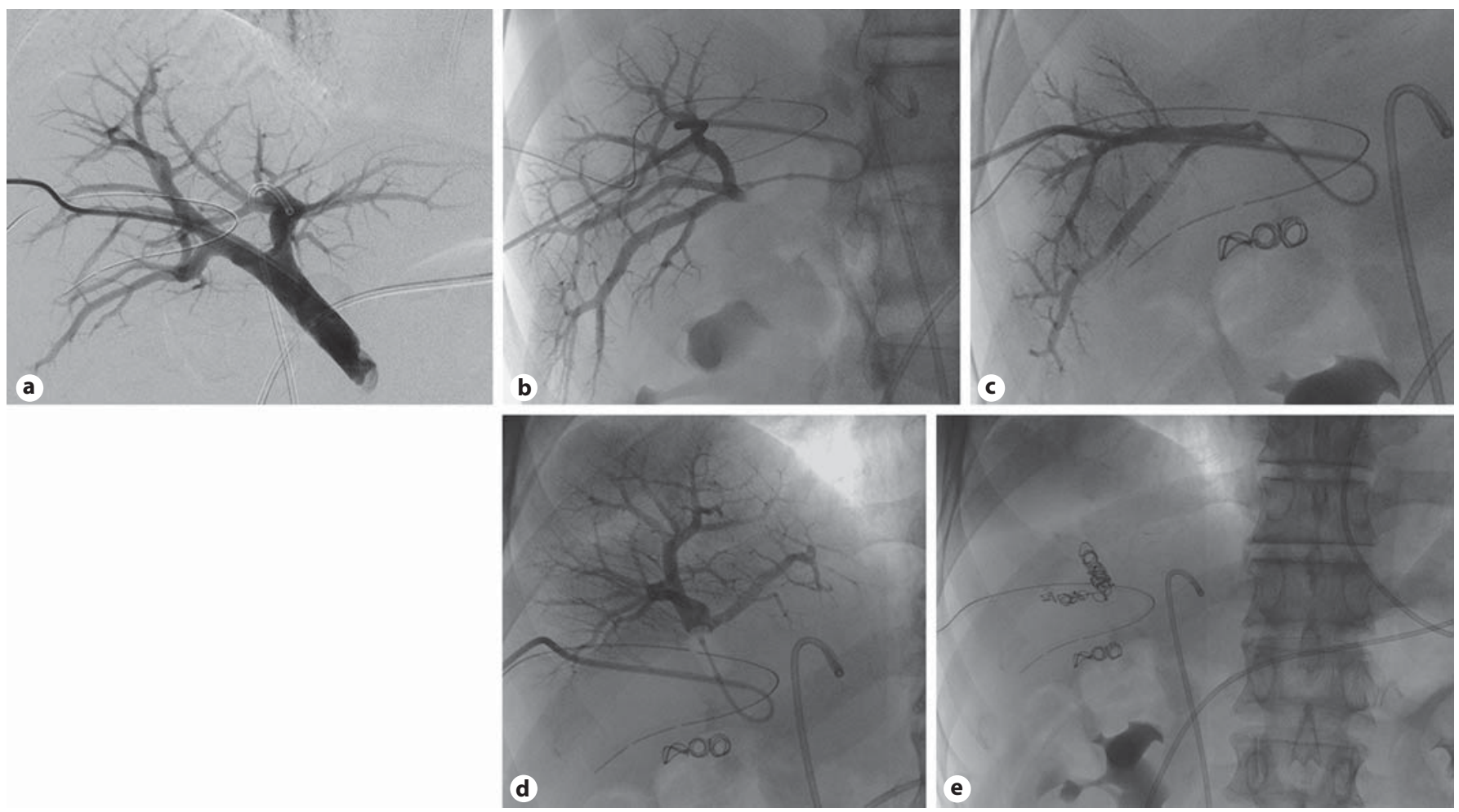

Fig. 1. Right PVE with a transhepatic ipsilateral approach. a Percutaneous transhepatic portogram indicates a usual ramification pattern of the portal system. An endoscopic and a percutaneous biliary drainage catheter are placed in the left hepatic duct and the right anterior sectorial duct, respectively. b Selective portogram of the right posterior sectorial branch (P6 + P7) using a re- verse-curved balloon catheter. Balloon occlusion can avoid dislodgement of steel coils as well as regurgitation of absolute ethanol. c Selective portogram of the right anterior inferior branch (P5). d Selective portogram of the right anterior superior branch (P8). e Completion of a right PVE. Steel coils are distally placed in the right anterior and posterior sectorial portal veins.

\section{Results}

\section{Portal Vein Embolization}

The actual number of embolized portal veins is summarized in table 1 . In the case of a right hepatectomy $(\mathrm{n}=318), 305$ patients underwent PVE of the right portal vein. The remaining 13 patients received a PVE of the right anterior $(\mathrm{n}=8)$ or posterior sectorial portal vein $(\mathrm{n}=5)$, owing to the obstruction of the right posterior or anterior portal vein by cancer, respectively. Of 54 patients who were to undergo a right trisectionectomy, 44 (81\%) underwent a right trisegment PVE, whereas the remaining 10 underwent a right PVE alone, mainly due to technical difficulty. In 121 patients who were to undergo a left trisectionectomy, 100 underwent a left trisegment PVE before surgery, whereas the remaining 21 underwent a PVE of the left portal vein $(n=5)$ or the right anterior portal vein $(n=16)$ due to an obstruction of the right anterior or the left portal vein by cancer, respectively.

Portal Vein Embolization for Biliary

Tract Cancer
Table 1. Type of planned hepatectomy and embolized portal vein

\begin{tabular}{llc}
\hline Planned hepatectomy & Embolized portal vein & Patients \\
\hline Right hepatectomy & Right & 305 \\
& Right anterior & 8 \\
& Right posterior & 5 \\
\hline Right trisectionectomy & Right and left medial & 44 \\
& Right & 10 \\
\hline Left trisectionectomy & Left and right anterior & 100 \\
& Left & 5 \\
& Right anterior & 16 \\
\hline Central bisectionectomy & Left medial and right anterior & 1 \\
\hline
\end{tabular}

\section{PVE-Related Complications}

A few patients developed an inflammatory response manifested by mild fever and/or mild abdominal pain or discomfort. Transaminase concentrations were ele- 
Fig. 2. Left trisegment PVE. a Portogram at supine position. Arrows indicate the site of steel coils placed. $\mathbf{b}$ Completion of a left trisegment PVE. The portal branches where steel coils were placed are denoted according to the nomenclature system of Couinaud and Takayasu.
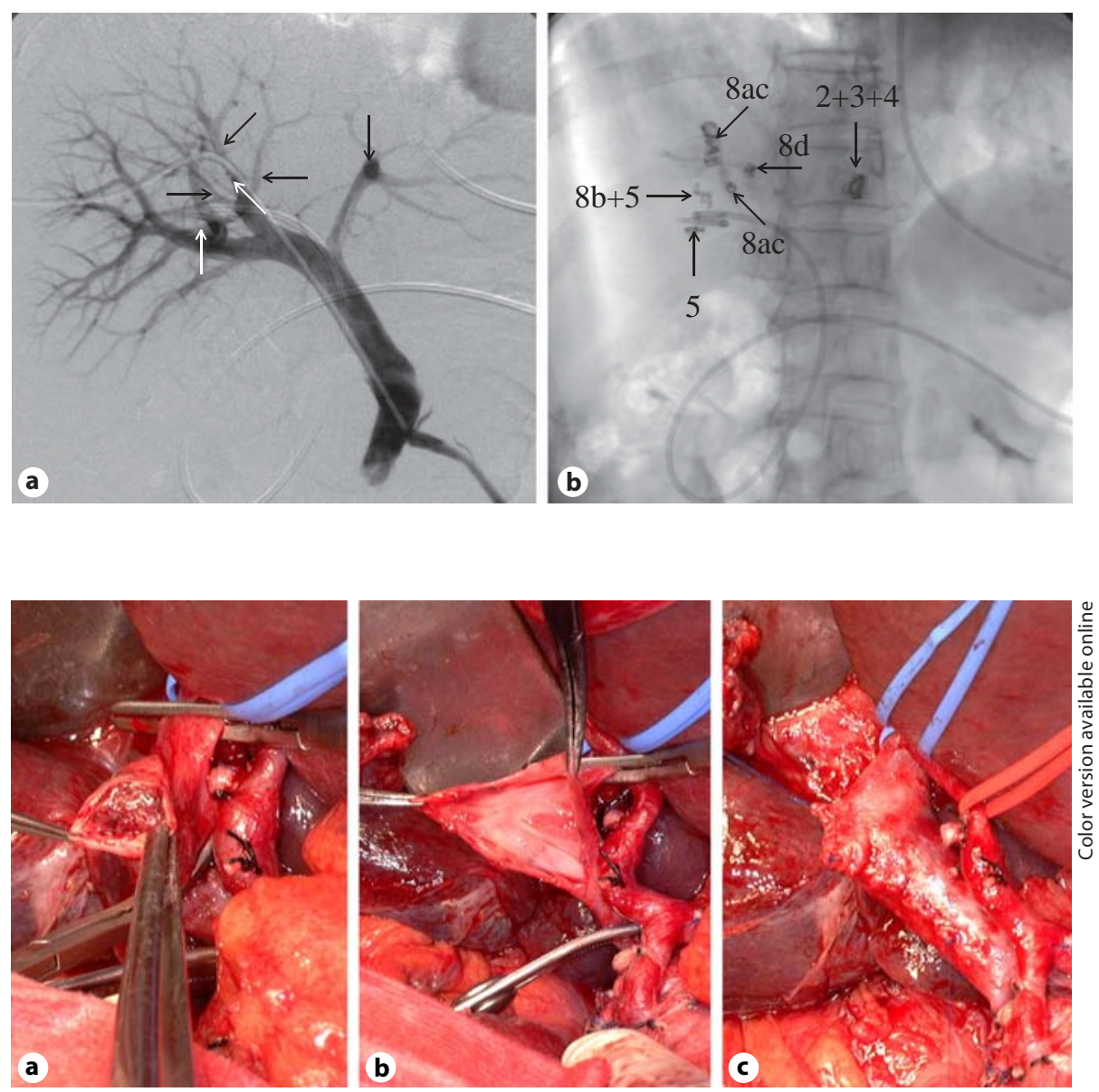

Fig. 3. PVE-induced thrombus at the stump of the right portal vein. a The right portal vein is divided with placement of cross clamps. b The thrombus is completely removed. c The stump is closed with continuous suture. vated, usually less than 3 times the normal range, which returned to baseline levels within a week for most patients. Serum total bilirubin remained near pre-embolization concentrations in all patients. Some complications occurred after PVE: extensive portal thrombosis requiring thrombolytic therapy, intra-abdominal bleeding requiring laparotomy, and ethanol-induced hemolysis requiring an administration of haptoglobin in one patient each. Overall, PVE-related complications requiring specific treatment were found in $3(0.6 \%)$ of the 494 patients, and no patients died of these complications.

During surgery, portal thrombus was occasionally found in the root of the embolized portal branch to be divided, hampering a simple ligation and division. In this situation, after cross-clamps were placed at the left portal vein and the main portal trunk, the thrombus was completely removed and then the stump was closed with continuous 5-0 prolene sutures (fig. 3 ).

\section{Surgical Outcome after PVE}

Of the 494 study patients, 122 (24.7\%) did not undergo subsequent hepatectomy because of peritoneal seeding, liver metastasis, periaortic node metastasis, and/or locally advanced disease. The unresectability rate after PVE was significantly higher in patients with gallbladder cancer than in those with cholangiocarcinoma: $43.2 \%$ $(61 / 141)$ versus $17.3 \%(61 / 353)$, respectively ( $p<0.001)$. The remaining 372 patients underwent hepatectomy (table 2). En bloc resection of the caudate lobe and the extrahepatic bile duct was performed in 368 (98.9\%) patients, combined pancreatoduodenectomy in 93 (25.0\%) patients, portal vein resection in $145(39.0 \%)$ patients, and hepatic artery resection in $41(11.0 \%)$ patients.

Of the 372 patients who underwent hepatectomy, 24 (6.5\%) died of postoperative complications. Surgical mortality was significantly higher in patients with gallbladder cancer than in those with cholangiocarcinoma: $16.3 \%$ $(13 / 80)$ versus $3.8 \%(11 / 292)$, respectively $(p<0.05)$. Before 2005 , mortality was $5.1 \%(7 / 136)$ in patients with 


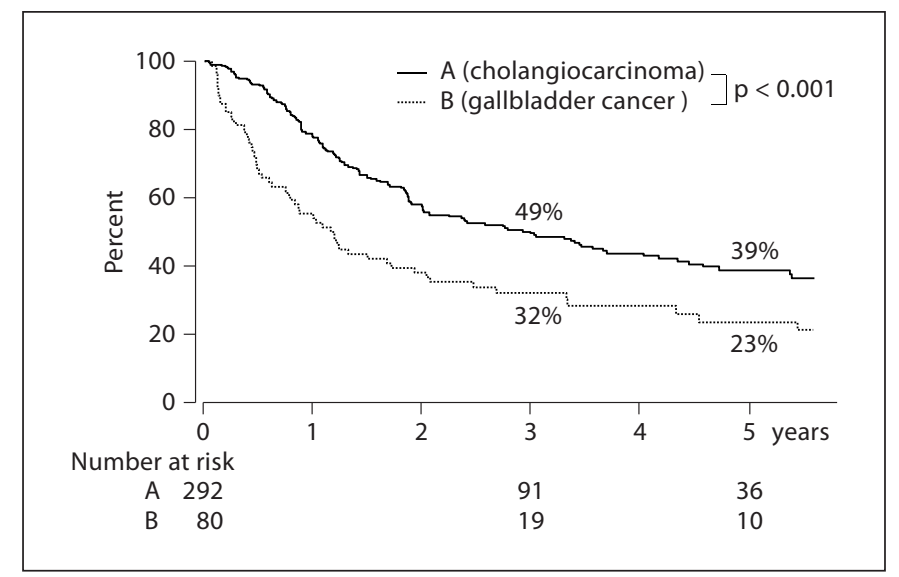

Fig. 4. Overall survival after extended hepatectomy following PVE. Statistical analysis by log-rank test.

cholangiocarcinoma and $19.2 \%$ (11/57) in those with gallbladder cancer; after 2006, mortality from these diseases was $2.5 \%(4 / 156)$ and $8.7 \%(2 / 23)$, respectively. Mortality significantly decreased from $9.3 \%$ (18/193) before 2005 to $3.4 \%(6 / 179)$ after $2006(\mathrm{p}<0.05)$.

\section{Staging and Survival}

Staging of the tumor is documented in table 3 according to the latest TNM classification of malignant tumors by the International Union against Cancer [17]. Most of the study patients had pT3 or pT4 tumors. Nodal metastasis was more common in patients with gallbladder cancer than in those with cholangiocarcinoma (60.0 and $42.5 \%$, respectively). Also, distant metastasis was more frequently observed in patients with gallbladder cancer than in those with cholangiocarcinoma (26.6 and 13.4\%, respectively). Overall, patients with gallbladder cancer had more advanced disease than those with cholangiocarcinoma.

The overall survival for patients with cholangiocarcinoma was significantly better than that for patients with gallbladder cancer (fig. 4), where the 3- and 5-year survival rate was 49 and $39 \%$ in the former group and 32 and $23 \%$ in the latter group, respectively $(\mathrm{p}<0.001)$. Thirtysix patients with cholangiocarcinoma and 10 patients with gallbladder cancer survived longer than 5 years after extended surgery. The survival of 227 patients with cholangiocarcinoma who underwent a less than 50\% resection of the liver (in whom preoperative PVE was not performed) during the same time period was almost identical to that of 292 patients with cholangiocarcinoma who underwent extended hepatectomy after PVE (fig. 5).

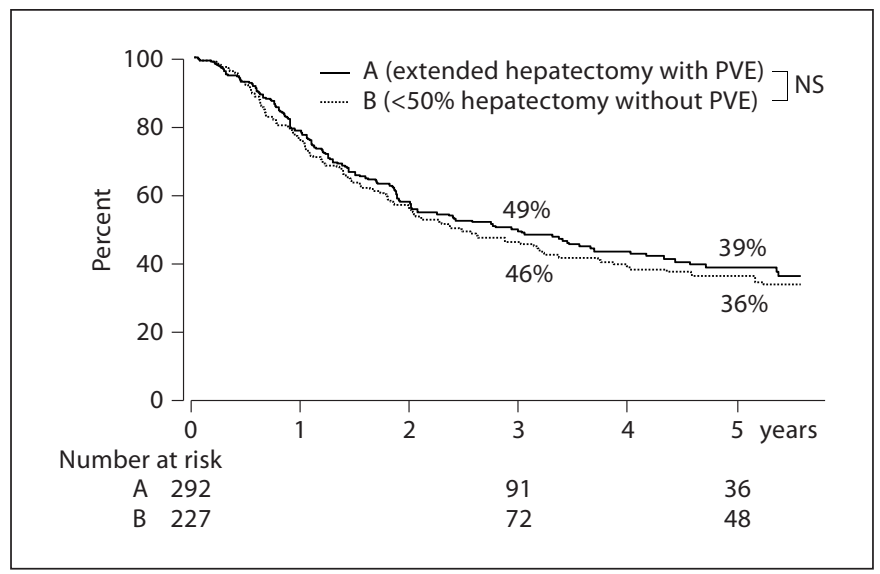

Fig. 5. Overall survival for patients with cholangiocarcinoma. Statistical analysis by log-rank test.

Table 2. Surgical procedures in 372 patients who underwent resection

\begin{tabular}{lccc}
\hline Procedures & Total & $\begin{array}{c}\text { Cholangio- } \\
\text { carcinoma }\end{array}$ & $\begin{array}{l}\text { Gallbladder } \\
\text { carcinoma }\end{array}$ \\
\hline Type of hepatectomy & & & \\
S1, 4, 5, 6, 7, 8 & $45(12 \%)$ & 38 & 7 \\
S1, 4b, 5, 6, 7, & $37(10 \%)$ & 1 & 36 \\
S1, 5, 6, 7, 8 & $149(50 \%)$ & 149 & 37 \\
S5, 6, 7, 8 & $4(1 \%)$ & 4 & 0 \\
S1, 2, 3, 4, 5, & $99(27 \%)$ & 99 & 0 \\
S1, 4, 5, 8 & $1(0.3 \%)$ & 1 & 0 \\
Combined resection & & & \\
Extrahepatic bile duct & $368(99 \%)$ & 288 & 80 \\
Pancreatoduodenectomy & $93(25 \%)$ & 60 & 33 \\
Portal vein & $145(39 \%)$ & 105 & 40 \\
$\quad$ Hepatic artery & $41(11 \%)$ & 41 & 0 \\
\hline
\end{tabular}

Table 3. Tumor node metastasis classification in 372 resected patients after PVE

\begin{tabular}{llll}
\hline & & $\begin{array}{l}\text { Cholangiocarcinoma } \\
(\mathrm{n}=292)\end{array}$ & $\begin{array}{l}\text { Gallbladder carcinoma } \\
(\mathrm{n}=80)\end{array}$ \\
\hline $\mathrm{pT}$ & 1 & $11(3.8 \%)$ & 0 \\
& 2 & $70(24.0 \%)$ & $4(5.0 \%)$ \\
& 3 & $61(20.9 \%)$ & $23(28.8 \%)$ \\
& 4 & $150(51.4 \%)$ & $53(66.2 \%)$ \\
$\mathrm{pN}$ & 0 & $168(57.5 \%)$ & $32(40.0 \%)$ \\
& 1 & $124(42.5 \%)$ & $48(60.0 \%)$ \\
\hline $\mathrm{pM}$ & 0 & $253(86.6 \%)$ & $51(63.4 \%)$ \\
& 1 & $39(13.4 \%)$ & $29(26.6 \%)$ \\
\hline
\end{tabular}




\section{Discussion}

Volume dynamics given by PVE should be taken into account when considering an indication of PVE. We previously reported a change in liver volume in 240 patients with biliary cancer who underwent PVE [13]. The volume of the non-embolized lobe significantly increased from $361 \mathrm{ml}$ before PVE to $460 \mathrm{ml}$ after PVE, and that of the embolized lobe significantly decreased from $688 \mathrm{ml}$ before PVE to $581 \mathrm{ml}$ after PVE. As for the volume ratio to the whole liver, the volume of the non-embolized lobe increased from $33 \%$ before PVE to $43 \%$ after PVE. Farges et al. [18] conducted a prospective study to assess the clinical benefit of PVE before a right hepatectomy, demonstrating that PVE has no beneficial effect in patients with a normal liver but significantly reduces postoperative complications in patients with underlying liver disease. A minimum essential volume of the future remnant is reported to be $25-30 \%$ in the normal liver [18-20]. Vauthey et al. [20] reported that major postoperative complications increased when the estimated liver remnant was less than $25 \%$. Recently, the ratio was lowered to $20 \%$ based on the 301 extended right hepatectomies, and it was concluded that a $20.1-30 \%$ future liver remnant is not an indication for preoperative PVE [21]. On the other hand, some authors stress that more than $40 \%$ of the total liver volume should be preserved to make the resection safe [22]. Taking these previous studies into consideration, PVE before major hepatectomy should be considered in patients with a damaged liver. In patients with a normal liver, PVE is essential when a planned procedure is complex or the estimated future remnant is less than $25-30 \%$. In our Department, PVE for biliary cancer is indicated when the estimated future liver remnant is less than $40 \%$ [13], although possible overutilization cannot be denied.

Belghiti et al. [23] analyzed the operative risk in 747 hepatectomized patients, and demonstrated that mortality in the case of a combined extrahepatic bile duct resection and in the case of jaundice was 21 and $5.8 \%$, respectively. This result indicates that hepatobiliary resection for biliary cancer involving the hepatic hilus is a risky procedure, and the functional capacity of the cholestatic liver should be independently assessed from that of the normal liver. In this study, $78 \%$ of the subjects had obstructive jaundice, all of the resected patients underwent extended hepatectomy, and $99 \%$ of the hepatectomized patients received extrahepatic bile duct resection. Considering these difficult settings, an overall mortality of $6.5 \%$ is acceptable. In this series, the functional capacity of the cholestatic liver was estimated by an integrated as- sessment of volume, as indicated by an estimated value of the indocyanine green clearance rate from the future liver remnant (remnant ICGK) $[13,24]$. We previously reported that remnant ICGK $\geq 0.05$ is a minimal requirement for safe hepatobiliary resection, and that PVE expands the proportion of patients with remnant ICGK $\geq 0.05$ from 45 to $86 \%$ [13]. As remnant ICGK has recently been strictly applied as an aid in decision-making, the mortality between 2006 and 2010 was 3.4\% (6/179) even after extended and difficult hepatectomy.

Despite the extent of remnant volume, PVE was often performed in some patients before complex hepatobiliary resection [25]. The embolized lobe after PVE is perfused with hepatic arterial flow alone. Therefore, temporary clamping of the proper hepatic artery yields a clear dark ischemic demarcation on the surface. In hepatectomy with combined vascular resection, inflow vascular division before liver transection is often impossible due to extensive tumor invasion at the hepatic hilus, so that ischemic demarcation cannot be obtained unless PVE is preceded. The right portal fissure between the right anterior and posterior sector, unlike the main or the umbilical fissure, cannot be accurately realized on the liver surface because of a lack of anatomic landmarks. Therefore, this temporary arterial clamping after PVE is useful in a left trisectionectomy with the simultaneous resection of the portal vein and hepatic artery for perihilar cholangiocarcinoma [26].

The survival for patients with cholangiocarcinoma was acceptable, while that of patients with advanced gallbladder cancer was poor. In addition, mortality after extended hepatectomy was significantly higher in patients with gallbladder cancer. The main reason for this poor outcome in gallbladder cancer is that combined vascular or multivisceral resections were performed more frequently in patients with gallbladder cancer. Twenty-seven percent of resected patients with gallbladder cancer had M1 disease, and most had hilar invasion. Although these patients may be considered unresectable in Western centers, 10 of 80 resected patients actually survived more than 5 years. Recently, we investigated the survival of patients with gallbladder cancer pathologically involving the extrahepatic duct [27]. The 5-year survival rate and median survival for 52 patients who survived right hepatectomy or more was $28 \%$ and 2.1 years, respectively, and all of these patients received preoperative PVE. These observations show a potential benefit of the aggressive surgery after PVE.

Abdalla et al. [28] stated in 2001 that 'a randomized control trial (RCT) cannot be recommended to test the efficacy of PVE, for it would be unethical to deny benefit 
of the technique and safer resection to patients who are otherwise poor candidates for resection based on inadequate liver size or function'. Although no RCTs have been conducted thereafter, we still agree with their statement, given the current level of evidence of PVE. The current study also confirms the same result reported in 2006 that PVE may benefit patients with advanced biliary cancer who are to undergo complicated, extended hepatectomy [13]. However, PVE is not omnipotent; therefore, further improvements in surgical technique and perioperative management is necessary to make difficult hepatectomy safer. Further prospective analyses of hepatic volume measurement and morbidity should help identify the population that appropriately benefits from PVE.

\section{References}

1 Kinoshita H, Sakai K, Hirohashi K, et al: Preoperative portal vein embolization for hepatocellular carcinoma. World J Surg 1986;10: 803-808.

2 Makuuchi M, Thai BL, Takayasu K, et al: Preoperative portal embolization to increase safety of major hepatectomy for hilar bile duct carcinoma: a preliminary report. Surgery 1990;107:521-527.

-3 Abulkhir A, Limongelli P, Healey AJ, et al: Preoperative portal vein embolization for major liver resection: a meta-analysis. Ann Surg 2008;247:49-57.

4 Nagino M, Nimura Y, Kamiya J, et al: Selective percutaneous transhepatic embolization of the portal vein in preparation for extensive liver resection: the ipsilateral approach. Radiology 1996;200:559-563.

5 Nagino M, Nimura Y, Kamiya J, et al: Right or left trisegment portal vein embolization before hepatic trisegmentectomy for hilar bile duct carcinoma. Surgery 1995;117:677681.

-6 Nagino M, Kamiya J, Kanai M, et al: Right trisegment portal vein embolization for biliary tract carcinoma: technique and clinical utility. Surgery 2000;127:155-160.

$\checkmark 7$ Madoff DC, Abdalla EK, Gupta S, et al: Transhepatic ipsilateral right portal vein embolization extended to segment IV: improving hypertrophy and resection outcomes with spherical particles and coils. J Vasc Interv Radiol 2005;16:215-225.

$\checkmark 8$ Nagino M, Nimura Y, Kamiya J, et al: Changes in hepatic lobe volume in biliary tract cancer patients after right portal vein embolization. Hepatology 1995;21:434-439.

-9 Uesaka K, Nimura Y, Nagino M: Changes in hepatic lobar function after right portal vein embolization. An appraisal by biliary indocyanine green excretion. Ann Surg 1996;223: 77-83.

10 Goto Y, Nagino M, Nimura Y: Doppler estimation of portal blood flow after percutaneous transhepatic portal vein embolization. Ann Surg 1998;228:209-213.
11 Nagino M, Nimura Y, Kamiya J, et al: Immediate increase in arterial blood flow in embolized hepatic segments after portal vein embolization: CT demonstration. AJR Am J Roentgenol 1998;171:1037-1039.

12 Kito Y, Nagino M, Nimura Y: Doppler sonography of hepatic arterial blood flow velocity after percutaneous transhepatic portal vein embolization. AJR Am J Roentgenol 2001;176:909-912.

13 Nagino M, Kamiya J, Nishio H, et al: Two hundred forty consecutive portal vein embolizations before extended hepatectomy for biliary cancer: surgical outcome and longterm follow-up. Ann Surg 2006;243:364372.

14 Nishio H, Nagino M, Kamiya J, et al: Most informative projection for portography: quantitative analysis of 47 percutaneous transhepatic portograms. World J Surg 2003; 27:433-436.

15 Shimamura T, Nakajima Y, Une Y, et al: Efficacy and safety of preoperative percutaneous transhepatic portal embolization with absolute ethanol: a clinical study. Surgery 1997;121:135-141.

16 Takayasu K, Moriyama N, Muramatsu Y, et al: Intrahepatic portal vein branches studied by percutaneous transhepatic portography. Radiology 1985;154:31-36.

17 Sobin LH, Gospodarowicz MK, Wittekind C: UICC TNM Classification of Malignant Tumours. New York, Wiley-Blackwell, 2009.

18 Farges O, Belghiti J, Kianmanesh R, et al: Portal vein embolization before right hepatectomy: prospective clinical trial. Ann Surg 2003;237:208-217.

19 Hemming AW, Reed AI, Howard RJ, et al: Preoperative portal vein embolization for extended hepatectomy. Ann Surg 2003;237: 686-691, discussion 691-693.
20 Vauthey JN, Chaoui A, Do KA, et al: Standardized measurement of the future liver remnant prior to extended liver resection: methodology and clinical associations. Surgery 2000;127:512-519.

21 Kishi Y, Abdalla EK, Chun YS, et al: Three hundred and one consecutive extended right hepatectomies: evaluation of outcome based on systematic liver volumetry. Ann Surg 2009;250:540-548.

22 Kubota K, Makuuchi M, Kusaka K, et al: Measurement of liver volume and hepatic functional reserve as a guide to decisionmaking in resectional surgery for hepatic tumors. Hepatology 1997;26:1176-1181.

23 Belghiti J, Hiramatsu K, Benoist S, et al: Seven hundred forty-seven hepatectomies in the 1990s: an update to evaluate the actual risk of liver resection. J Am Coll Surg 2000;191: 38-46.

24 Yokoyama Y, Nishio H, Ebata T, et al: Value of indocyanine green clearance of the future liver remnant in predicting outcome after resection for biliary cancer. Br J Surg 2010;97: 1260-1268.

25 Igami T, Yokoyama Y, Nishio H, et al: A left hepatectomy and caudate lobectomy combined resection of the ventral segment of the right anterior sector for hilar cholangiocarcinoma - the efficacy of PVE (portal vein embolization) in identifying the hepatic subsegment: report of a case. Surg Today 2009; 39:628-632.

26 Nagino M, Nimura Y, Nishio H, et al: Hepatectomy with simultaneous resection of the portal vein and hepatic artery for advanced perihilar cholangiocarcinoma: an audit of 50 consecutive cases. Ann Surg 2010;252:115123.

27 Nishio H, Ebata T, Yokoyama Y, et al: Gallbladder cancer involving the extrahepatic bile duct is worthy of resection. Ann Surg 2011;253:953-960.

28 Abdalla EK, Hicks ME, Vauthey JN: Portal vein embolization: rationale, technique and future prospects. Br J Surg 2001;88:165-175. 